\title{
DERIVATIONS ON BANACH ALGEBRAS
}

\section{S. HEJAZIAN and S. TALEBI}

\author{
Received 12 September 2002
}

\begin{abstract}
Let $D$ be a derivation on a Banach algebra; by using the operator $D^{2}$, we give necessary and sufficient conditions for the separating ideal of $D$ to be nilpotent. We also introduce an ideal $M(D)$ and apply it to find out more equivalent conditions for the continuity of $D$ and for nilpotency of its separating ideal.
\end{abstract}

2000 Mathematics Subject Classification: 46H40, 47B47.

1. Introduction. Let $A$ be a Banach algebra. By a derivation on $A$, we mean a linear mapping $D: A \rightarrow A$, which satisfies $D(a b)=a D(b)+D(a) b$ for all $a$ and $b$ in $A$. The separating space of $D$ is the set

$$
S(D)=\left\{a \in A: \exists\left\{a_{n}\right\} \subset A ; a_{n} \longrightarrow 0, D\left(a_{n}\right) \longrightarrow a\right\} .
$$

The set $S(D)$ is a closed ideal of $A$ which, by the closed-graph theorem, is zero if and only if $D$ is continuous.

DEFINITION 1.1. A closed ideal $J$ of $A$ is said to be a separating ideal if, for each sequence $\left\{a_{n}\right\}$ in $A$, there is a natural $N$ such that

$$
\overline{\left(J a_{n} \cdots a_{1}\right)}=\overline{\left(J a_{N} \cdots a_{1}\right)} \quad(n \geq N) .
$$

The separating space of a derivation on $A$ is a separating ideal [2, Chapter 5]; it also satisfies the same property for the left products.

The following assertions are of the most famous conjectures about derivations on Banach algebras:

(C1) every derivation on a Banach algebra has a nilpotent separating ideal;

(C2) every derivation on a semiprime Banach algebra is continuous;

(C3) every derivation on a prime Banach algebra is continuous;

(C4) every derivation on a Banach algebra leaves each primitive ideal invariant.

Clearly, if (C1) is true, then the same for (C2) and (C3). Mathieu and Runde in [5] proved that (C1), (C2), and (C3) are equivalent. The conjecture (C4) is known as the noncommutative Singer-Wermer conjecture, and it has been proved in [1] that if each of the conjectures (C1), (C2), or (C3) hold, then (C4) is also true. The conjectures (C1), (C2), and (C3) are still open even if $A$ is assumed 
to be commutative, but (C4) is true in the commutative case, see [7]. These conjectures are also related to some other famous open problems; the reader is referred to $[1,3,4,5,9]$ for more details.

In the next section, we deal with (C1), and although, for a derivation $D$ on a Banach algebra, the operators $D^{n}, n=2,3, \ldots$, are more complicated, by considering $D^{2}$, we easily give some equivalent conditions for $S(D)$ to be nilpotent. As a consequence, we reprove some of the results in [8]. At the end of the next section, we introduce an ideal related to a derivation and apply it to obtain some equivalent conditions for continuity of $D$ and for nilpotency of $S(D)$.

We recall that $S(D)$ is nilpotent if and only if $S(D) \cap R$ is nilpotent, see [1, Lemma 4.2].

2. The results. From now on, $A$ is a Banach algebra, and $R$ and $L$ denote the Jacobson radical and the nil radical of $A$, respectively, (see [6, Chapter 4] for definitions). Note that $D$ is a derivation on $A$, and $S(D)$ is the separating ideal of $D$. If $B_{i}$ 's, $i=1,2, \ldots, n$, are subsets of $A$, then $B_{1} B_{2} \cdots B_{n}$ denotes the linear span of the set $\left\{b_{1} b_{2} \cdots b_{n}: b_{i} \in B_{i}\right.$, for $\left.i=1,2, \ldots, n\right\}$, and if all of $B_{i}$ 's coincide with each other, we denote this set by $B^{n}$.

THEOREM 2.1. Let $J$ be a closed left ideal of $A$. Then, $S(D) \cap J$ is nilpotent if and only if $\left.D^{2}\right|_{\overline{\bigcap_{n=1}^{\infty}(S(D) \cap J)^{n}}}$ is continuous.

Proof. Suppose that $D^{2}$ is continuous on $\overline{\bigcap_{n=1}^{\infty}(S(D) \cap J)^{n}}$. Consider $a$ in $S(D) \cap J$, then for each $n \in \mathbb{N}, a^{n} \in(S(D) \cap J)^{n}$, and since $S(D)$ is a separating ideal, there exists $N \in \mathbb{N}$ such that

$$
\overline{S(D) a^{n}}=\overline{S(D) a^{N}} \quad(n \geq N) .
$$

Hence, by the Mittag-Leffler theorem [2, Theorem A.1.25] and the fact that $S(D) a^{n} \subseteq(S(D) \cap J)^{n}$, we have

$$
\overline{S(D) a^{N}}=\bigcap_{n=1}^{\infty} \overline{S(D) a^{n}}=\overline{\bigcap_{n=1}^{\infty} S(D) a^{n}} \subseteq \overline{\bigcap_{n=1}^{\infty}(S(D) \cap J)^{n}}
$$

Now, let $\left\{x_{n}\right\} \subseteq A, x_{n} \rightarrow 0$, and $D\left(x_{n}\right) \rightarrow a^{N+1}$. Take $y_{n}=x_{n} a^{N+1}$, then $y_{n} \in S(D) a^{N} \subseteq \overline{\bigcap_{n=1}^{\infty}(S(D) \cap J)^{n}}, y_{n} \rightarrow 0$, and $D\left(y_{n}\right) \rightarrow a^{2(N+1)}$, and by the hypothesis, $D^{2}\left(y_{n}\right) \rightarrow 0$ and $D^{2}\left(y_{n}{ }^{2}\right) \rightarrow 0$. On the other hand,

$$
D^{2}\left(y_{n}^{2}\right)=y_{n} D^{2}\left(y_{n}\right)+2\left(D y_{n}\right)^{2}+D^{2}\left(y_{n}\right) y_{n} \longrightarrow 2 a^{4(N+1)}
$$

Therefore, $a^{4 N+4}=0$, that is, $S(D) \cap J$ is a nil and hence a nilpotent ideal by closedness [6, Theorem 4.4.11]. The converse is trivial. 
REMARK 2.2. (i) Note that in Theorem 2.1, we can replace $J$ by a right ideal, see [2, Theorem 5.2.24].

(ii) The argument of Theorem 2.1 shows that if $J$ is not assumed to be closed and if $D^{2}$ is continuous on $\overline{\bigcap_{n=1}^{\infty}(S(D) \cap J)^{n}}$, then $S(D) \cap J$ will be a nil ideal.

COROLLARY 2.3. The set $S(D)$ is nilpotent if and only if $\left.D^{2}\right|_{\overline{\bigcap_{n=1}^{\infty}(S(D) \cap R)^{n}}}$ is continuous.

Proof. If $S(D)$ is nilpotent, then the result is obvious. Conversely, by Theorem 2.1, $S(D) \cap R$ is nilpotent, and by [1, Lemma 4.2], $S(D)$ is nilpotent.

COROLLARY 2.4. If $\operatorname{dim}\left(\bigcap_{n=1}^{\infty}(S(D) \cap R)^{n}\right)<\infty$, then $S(D)$ is nilpotent.

The assertions of the following theorem were proved by Villena in [8], see also [9, Theorem 4.4]. Using Theorem 2.1, we can reprove them in a different way.

THEOREM 2.5. The derivation $D$ is continuous if one of the following assertions hold:

(a) $A$ is semiprime and $\operatorname{dim}\left(R \cap\left(\cap_{n=1}^{\infty} A^{n}\right)\right)<\infty$;

(b) $A$ is prime and $\operatorname{dim}\left(\bigcap_{n=1}^{\infty}(a A \cap R)^{n}\right)<\infty$ for some $a \in A$ with $a^{2} \neq 0$;

(c) $A$ is an integral domain and $\operatorname{dim}\left(\cap_{n=1}^{\infty}(a A \cap R)^{n}\right)<\infty$ for some nonzero $a \in A$.

Proof. (a) By Corollary 2.4, $S(D)$ is nilpotent, and since $A$ is semiprime, $D$ is continuous.

(b) Without loss of generality, we may assume that $A$ has an identity. By assumption, $\bigcap_{n=1}^{\infty}(a A \cap R \cap S(D))^{n}$ is finite dimensional; thus, $D^{2}$ is continuous on this space, and by Remark 2.2(ii), $a A \cap R \cap S(D)$ is a nil right ideal; therefore, $a(S(D) \cap R)$ is a nil right ideal, and by [6, Theorem 4.4.11], $a(S(D) \cap R) \subseteq L=$ $\{0\}$. Thus, $\operatorname{AaA}(S(D) \cap R)=\{0\}$, where $A a A$ is the ideal generated by $a$. Since $a^{2} \neq 0$ and $A$ is prime, it follows that $S(D) \cap R=\{0\}$ and hence $S(D) \subseteq L=\{0\}$.

(c) The same argument as in (b) shows that $a(S(D) \cap R)=\{0\}$, and since $A$ is an integral domain, $S(D) \cap R=\{0\}$ and $D$ is continuous.

In the sequel, we give other equivalent conditions for $S(D)$ to be nilpotent, but first we introduce the set

$$
M(D)=\{x \in S(D) \cap R: D(x) \in R\} .
$$

Obviously, $M(D)$ is an ideal of $A$ and $(S(D) \cap R)^{2} \subseteq M(D)$. The following theorems show that this ideal can help us to study the continuity of a derivation or nilpotency of its separating ideal.

THEOREM 2.6. The derivation $D$ is continuous if and only if $M(D)=\{0\}$. 
Proof. Clearly, if $D$ is continuous, then $M(D)=\{0\}$. Conversely, let $M(D)=$ $\{0\}$; then, $(S(D) \cap R)^{2}=\{0\}$. Therefore, $(S(D) \cap R)$ and hence $S(D)$ is a nilpotent ideal. Therefore, $S(D) \subseteq L$; we also have $D(L) \subseteq L$ by [1, Lemma 4.1]; thus, $D(S(D)) \subseteq R$, that is, $S(D) \subseteq M(D)=\{0\}$ and $D$ is continuous.

THEOREM 2.7. The following assertions are equivalent:

(a) $S(D)$ is nilpotent;

(b) $M(D)$ is a nil ideal;

(c) $\bigcap_{n=1}^{\infty} M(D)^{n}=\{0\}$.

Proof. Clearly, (a) implies (b). Suppose that (b) holds, then $(S(D) \cap R)^{2}$ is a nil ideal; therefore, $S(D)$ is a nilpotent ideal and (a) holds. Now, if $S(D)$ is nilpotent, then $\bigcap_{n=1}^{\infty}\left(S(D)^{n}\right)=\{0\}$ and this implies (c). Finally, if $\bigcap_{n=1}^{\infty} M(D)^{n}=\{0\}$, then by Theorem 2.1 and Remark 2.2 $M(D)=M(D) \cap S(D)$ is a nil ideal and (c) implies (b).

Acknowledgment. The authors would like to thank The Payame Noor University of Iran for the financial support.

\section{REFERENCES}

[1] J. Cusack, Automatic continuity and topologically simple radical Banach algebras, J. London Math. Soc. (2) 16 (1977), no. 3, 493-500.

[2] H. G. Dales, Banach Algebras and Automatic Continuity, London Mathematical Society Monographs. New Series, vol. 24, The Clarendon Press, New York, 2000.

[3] M. Mathieu, Where to find the image of a derivation, Functional Analysis and Operator Theory (Warsaw, 1992), Banach Center Publ., vol. 30, Polish Academy of Sciences, Warsaw, 1994, pp. 237-249.

[4] M. Mathieu and G. J. Murphy, Derivations mapping into the radical, Arch. Math. (Basel) 57 (1991), no. 5, 469-474.

[5] M. Mathieu and V. Runde, Derivations mapping into the radical. II, Bull. London Math. Soc. 24 (1992), no. 5, 485-487.

[6] T. W. Palmer, Banach Algebras and the General Theory of *-Algebras. Vol. I, Encyclopedia of Mathematics and Its Applications, vol. 49, Cambridge University Press, Cambridge, 1994.

[7] M. P. Thomas, The image of a derivation is contained in the radical, Ann. of Math. (2) 128 (1988), no. 3, 435-460.

[8] A. R. Villena, Derivations with a hereditary domain. II, Studia Math. 130 (1998), no. 3, 275-291.

[9] - Automatic continuity in associative and nonassociative context, Irish Math. Soc. Bull. (2001), no. 46, 43-76.

S. Hejazian: Department of Mathematics, Ferdowsi University, Mashhad, Iran

E-mail address: hejazian@math.um.ac.ir

S. Talebi: Department of Mathematics, Payame Noor University, Mashhad, Iran

E-mail address: talebi@mshc. pnu.ac. ir 


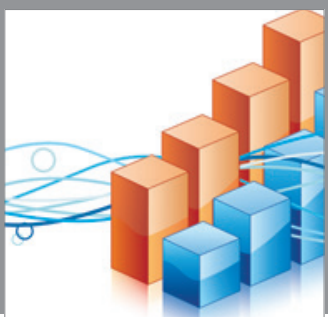

Advances in

Operations Research

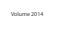

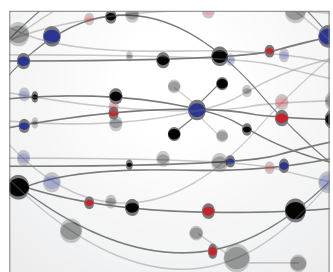

\section{The Scientific} World Journal
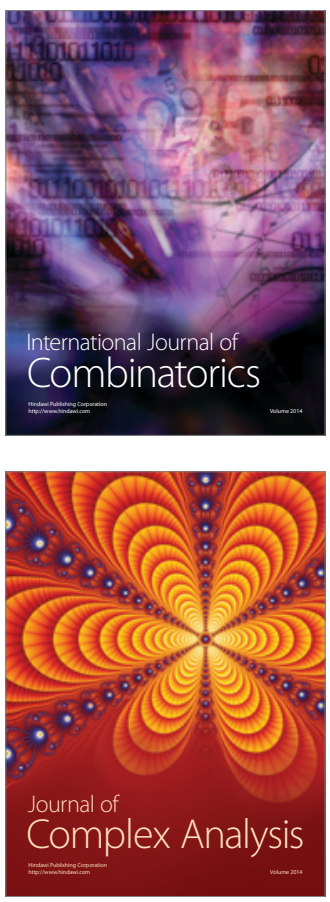

International Journal of

Mathematics and

Mathematical

Sciences
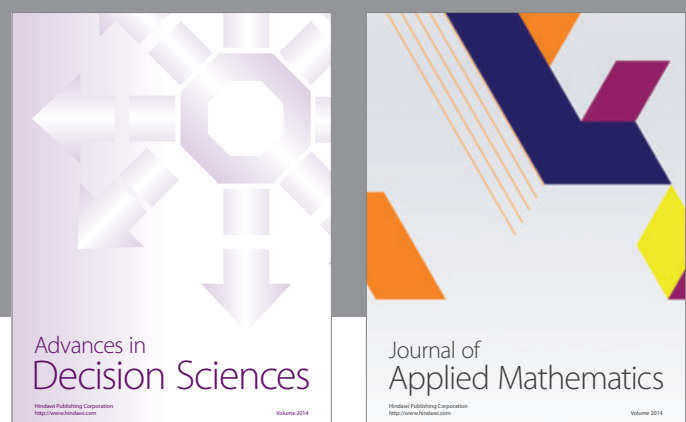

Journal of

Applied Mathematics
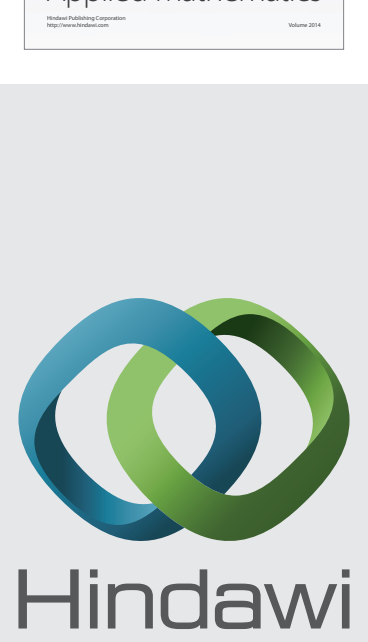

Submit your manuscripts at http://www.hindawi.com
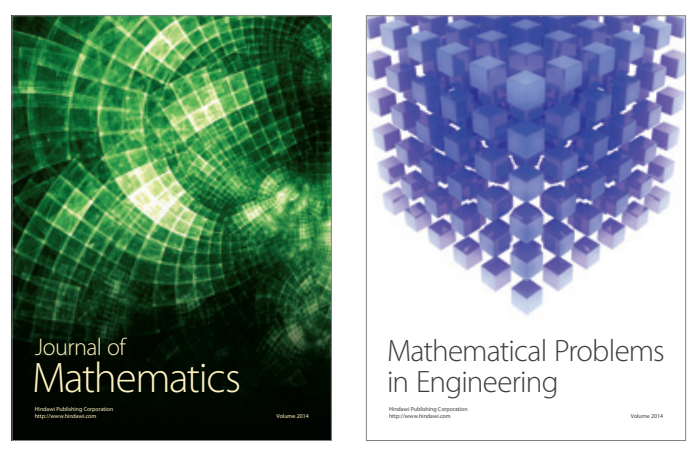

Mathematical Problems in Engineering
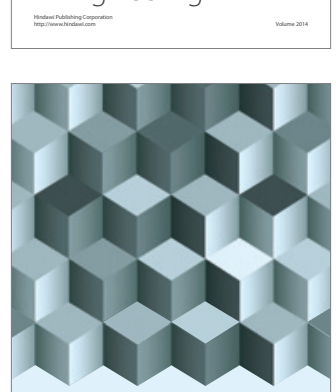

Journal of

Function Spaces
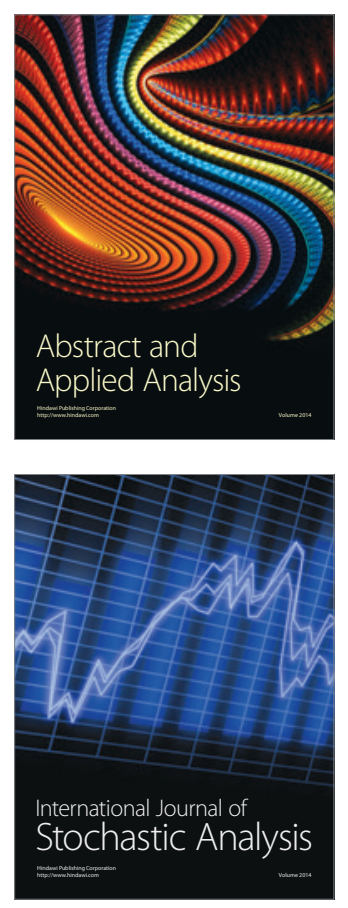

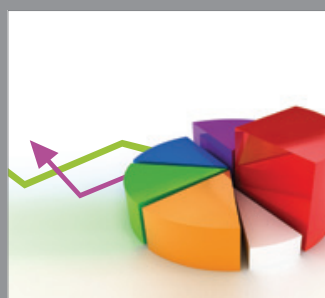

ournal of

Probability and Statistics

Promensencen
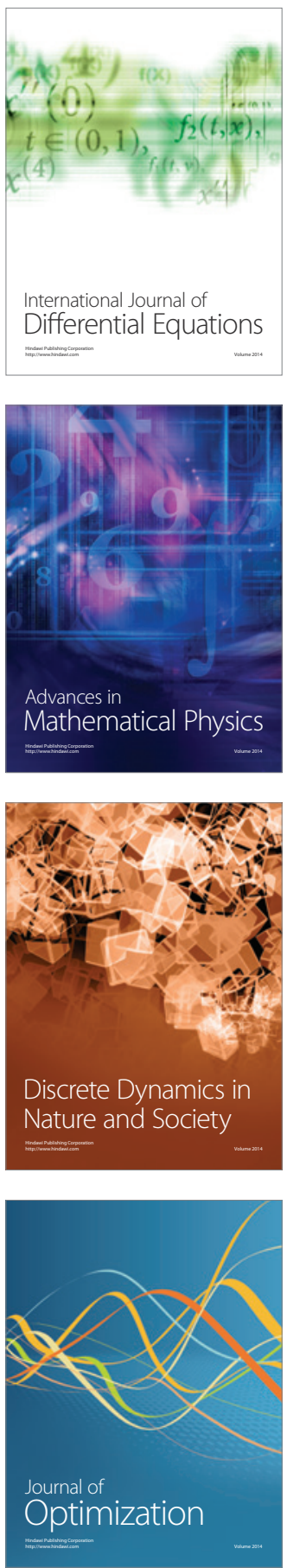